\title{
On the susceptibility of bright nonlinear Schrödinger solitons to long-wave transverse instability
}

\author{
By ThOMAS J. BRIDGES \\ Department of Mathematics and Statistics, University of Surrey, \\ Guildford, Surrey GU2 7XH, UK (t.bridges@eim.surrey.ac.uk) \\ Received 12 November 2003; revised 1 March 2004; accepted 30 March 2004; \\ published online 2 June 2004
}

A new theory for transverse instability of bright solitons of equations of nonlinear Schrödinger (NLS) type is presented, based on a natural deformation of the solitons into a four-parameter family. This deformation induces a set of four diagnostic functionals which encode information about transverse instability. These functionals include the deformed power, the deformed momentum and two new functionals. The main result is that a sufficient condition for long-wave transverse instability is completely determined by these functionals. Whereas longitudinal instability is determined by a single partial derivative (the Vakhitov-Kolokolov criterion), the condition for transverse instability requires 10 partial derivatives. The theory is illustrated by application to scalar NLS equations with general potential, and vector NLS equations for optical media with $\chi^{(2)}$ nonlinearity.

Keywords: solitary waves; optical media; transverse instability; multi-symplectic; nonlinear Schrödinger equation; Hamiltonian

\section{Introduction}

The transverse instability of bright solitary waves (BSWs) of models based on single or coupled nonlinear Schrödinger (NLS) equations is of fundamental importance in oceanic, optical and other applications. BSWs are localized in space and have harmonic time dependence. Transverse instability is of interest because it provides a mechanism for the development of multi-dimensional spatially localized coherent structures. The recent review paper of Kivshar \& Pelinovsky (2000) has a history and an extensive list of references for transverse instability of BSWs of NLS-type models.

The longitudinal stability and instability of BSWs has been even more widely studied. One of the fundamental results on longitudinal stability for BSWs of the scalar NLS equation is the Vakhitov-Kolokolov (VK) criterion for stability. Let $\mathcal{P}$ be the power of the BSW and let $\omega$ be the frequency of the harmonic time dependence. Then the VK condition is

$$
\frac{\partial \mathcal{P}}{\partial \omega}<0 \Rightarrow \text { instability }
$$


and

$$
\frac{\partial \mathcal{P}}{\partial \omega}>0 \Rightarrow \text { stability }
$$

This result was discovered by Vakhitov \& Kolokolov (1973) and has been rigorously justified by Weinstein (1986) and Grillakis et al. (1987). The VK condition is useful in applications as it uses only information available from the existence of a BSW to predict instability. It is important to note that the VK condition applies only to systems for which the second variation has a single negative eigenvalue (see Weinstein (1986) and Grillakis et al. (1987) for detailed discussion). The main example for which the VK condition applies conclusively is the scalar NLS model. But it is generally not conclusive for vector NLS models (see discussion in $\S 4$ ).

A generalization of the VK condition to transverse instability of BSWs has not been found, heretofore. It is the purpose of this paper to show that there is a generalization of the VK condition to transverse instability, and it does not require any information from the second variation. This result is surprising for two reasons. First, the BSWs are in a one-parameter family, and therefore there is no more information than the frequency dependence of the power. In fact there is a hidden dependence on three more parameters: classical BSWs can be interpreted as a one-parameter slice through a four-parameter family of BSWs. Second, information from the second variation is not required.

Deforming the BSWs into this four-parameter family leads to several new results. First, a geometric condition for instability generalizing the VK criterion is found. Second, the deformation provides information about the susceptibility of BSWs to transverse instability. Third, the theory proposes two new diagnostic functionals whose measurement is of independent experimental interest. Fourth, the four-parameter deformation of BSWs may be of independent theoretical interest as a larger class of BSWs.

The geometric condition for transverse instability is a sufficient condition for longwave transverse instability. The converse - when all roots of $\Delta(\Omega)$ defined below are real-is a necessary but not sufficient condition for transverse stability. When all roots of $\Delta(\Omega)$ are real, there are two ways that instability can still arise: either through short-wave transverse instability, or through long-wave instability with $\operatorname{Re}(\lambda)$ of finite amplitude. This latter case arises if the wave is longitudinally unstable and this instability persists for small transverse wavenumber.

The theory developed here applies to any basic state which is localized in space and periodic in time (with the periodicity associated with symmetry) and generalities will be discussed at the end of the paper, but for illustration of the theory two examples will be used: an NLS model with general potential $F(\cdot)$,

$$
\mathrm{i} \psi_{t}+\psi_{x x}+\sigma \psi_{y y}+F^{\prime}\left(|\psi|^{2}\right) \psi=0, \quad \sigma= \pm 1 ;
$$

and an NLS model for optical media with $\chi^{(2)}$, or mixed $\chi^{(2)}-\chi^{(3)}$, nonlinearity

$$
\left.\begin{array}{r}
\mathrm{i} \psi_{t}+\psi_{x x}+\sigma_{1} \psi_{y y}+\bar{\psi} \varphi+s\left(|\psi|^{2}+\rho|\varphi|^{2}\right) \psi=0, \\
2 \mathrm{i} \varphi_{t}+\varphi_{x x}+\sigma_{2} \varphi_{y y}+\frac{1}{2} \psi^{2}+s\left(\eta|\varphi|^{2}+\rho|\psi|^{2}\right) \varphi=0 .
\end{array}\right\}
$$

Proc. R. Soc. Lond. A (2004) 
Details of this model and definitions of the constants can be found in Bang et al. (1998). Classical BSWs are $y$-independent solutions of the form

$$
\psi(x, t)=\mathrm{e}^{\mathrm{i} \omega t} u(x), \quad \varphi(x, t)=\mathrm{e}^{2 \mathrm{i} \omega t} v(x)
$$

(or just $\psi(x, t)$ for (1.1)), where $u(x)$ and $v(x)$ are real-valued localized functions.

The strategy is to test the susceptibility of solitons in this class to transverse instability by deforming (embedding) the basic state into a multi-parameter family. This deformation will induce a set of diagnostic functionals. To illustrate the basics of the theory, the model (1.1) will be used.

Deform (1.3) for (1.1) into the following four-parameter family of solitons:

$$
\psi(x, y, t)=\mathrm{e}^{\mathrm{i} \theta} u(\xi),
$$

where

$$
\theta=\omega t+a y+\theta_{0} \quad \text { and } \quad \xi=x+\ell y+b t+\xi_{0} .
$$

The function $u(\xi)$ is now, in general, complex valued, and $\theta_{0}$ and $\xi_{0}$ are arbitrary phase shifts. There are three additional parameters, $\boldsymbol{p}:=(a, b, \ell)$, and in the limit $\boldsymbol{p} \rightarrow 0$, the deformed solitary wave (1.4) reduces to the classical bright solitary wave (1.3). For (1.1) one can show explicitly that the BSW persists in the deformed family (subject to natural restrictions on the parameters). The justification for this particular deformation is realized when analysing the linear stability problem: some inhomogeneous problems can be clearly solved explicitly after this deformation; see equation (3.2) and the discussion thereafter.

For classical BSWs, a strategy is to deform (1.3) into (1.4), evaluate (1.4) on a set of diagnostic functionals, and then reverse deform, $\boldsymbol{p} \rightarrow 0$, to the classical BSW.

In $\S 2$, it is shown that this four-parameter deformation induces a set of four diagnostic functionals

$$
\left.\begin{array}{rl}
\mathcal{P} & =\oint \operatorname{Im}\left(\bar{\psi} \psi_{\theta}\right) \mathrm{d} \xi \mathrm{d} \theta, \\
\mathcal{A} & =\oint\left[2 \sigma a\left|\psi_{\theta}\right|^{2}+2 \sigma \ell \operatorname{Re}\left(\bar{\psi}_{\theta} \psi_{\xi}\right)\right] \mathrm{d} \xi \mathrm{d} \theta, \\
\mathcal{B} & =\oint \operatorname{Im}\left(\bar{\psi} \psi_{\xi}\right) \mathrm{d} \xi \mathrm{d} \theta, \\
\mathcal{C} & =\oint\left[2 \sigma \ell\left|\psi_{\xi}\right|^{2}+2 \sigma a \operatorname{Re}\left(\bar{\psi}_{\theta} \psi_{\xi}\right)\right] \mathrm{d} \xi \mathrm{d} \theta,
\end{array}\right\}
$$

where $\oint$ represents the integral

$$
\oint(\cdot) \mathrm{d} \xi \mathrm{d} \theta=\frac{1}{2 \pi} \int_{0}^{2 \pi} \int_{-\infty}^{+\infty}(\cdot) \mathrm{d} \xi \mathrm{d} \theta .
$$

With $\psi(x, t)$ of the form (1.3), the first functional $\mathcal{P}$ is the familiar power function, and is the conserved quantity associated with the $S O(2)$ symmetry of (1.1). The third functional $\mathcal{B}$ is related to the deformed horizontal ( $x$-direction) momentum. The other two functionals, $\mathcal{A}$ and $\mathcal{C}$, are new. They are critical for determining susceptibility to transverse instability. They are discovered naturally after a multi-symplectification of (1.1). It would be of interest to identify their physical significance. 
These four functionals arise in the prediction of transverse instability of (1.3) as follows. It is shown in $\S 3$ that all long-wave (small transverse wavenumbers) instabilities for (1.1) or (1.2) are determined by a quartic polynomial, $\Delta(\Omega)=0$, where $\Omega$ is related to the stability exponent, and

$$
\Delta(\Omega)=\operatorname{det}\left[\left(\begin{array}{cc}
\mathcal{P}_{\omega} & \mathcal{P}_{b} \\
\mathcal{B}_{\omega} & \mathcal{B}_{b}
\end{array}\right) \Omega^{2}+\left(\begin{array}{cc}
\mathcal{P}_{a}+\mathcal{A}_{\omega} & \mathcal{P}_{\ell}+\mathcal{A}_{b} \\
\mathcal{B}_{a}+\mathcal{C}_{\omega} & \mathcal{B}_{\ell}+\mathcal{C}_{b}
\end{array}\right) \Omega+\left(\begin{array}{cc}
\mathcal{A}_{a} & \mathcal{A}_{\ell} \\
\mathcal{C}_{a} & \mathcal{C}_{\ell}
\end{array}\right)\right]
$$

A purely imaginary root of $\Delta(\Omega)=0$ indicates instability. The justification for this instability condition is in $\S 3$. It is remarkable that longitudinal instability requires only one derivative, $\mathcal{P}_{\omega}$, whereas prediction of transverse instability requires 10 derivatives! Details of the derivation of this stability quartic are given in $\S 3$. This theory generalizes the geometric criterion of Bridges (2000) for transverse instability of classical steady solitary waves (i.e. without the harmonic time dependence), where only three derivatives are needed.

The above stability polynomial gives long-wave transverse instability for any member of the four-parameter family. It simplifies dramatically if one is interested in the classical $(\boldsymbol{p}=0)$ BSW. In the limit $\boldsymbol{p} \rightarrow 0$, the stability quartic factorizes to

$$
\frac{\partial \mathcal{P}}{\partial \omega} \Omega^{2}=-\frac{\partial \mathcal{A}}{\partial a} \text { and } \frac{\partial \mathcal{B}}{\partial b} \Omega^{2}=-\frac{\partial \mathcal{C}}{\partial \ell} .
$$

The generalization of the VK condition to transverse instability of classical BSWs is immediate. Suppose there exists a solitary wave solution of (1.1) of the form (1.4). Then in the limit $\boldsymbol{p} \rightarrow 0$, we find that the wave is transverse unstable if either

$$
\frac{\partial \mathcal{P}}{\partial \omega} \frac{\partial \mathcal{A}}{\partial a}>0 \quad \text { or } \quad \frac{\partial \mathcal{B}}{\partial b} \frac{\partial \mathcal{C}}{\partial \ell}>0 .
$$

This result applies to both (1.1) and (1.2), as shown in $\S 4$ for the latter. For (1.1) the diagnostic functionals (for any $F(\cdot)$ for which a BSW exists) reduce to

$$
\left.\begin{array}{ll}
\mathcal{P}=\|v\|_{2}^{2}, & \mathcal{A}=2 \sigma(a+\ell \kappa(\boldsymbol{p}))\|v\|_{2}^{2}, \\
\mathcal{B}=\kappa(\boldsymbol{p})\|v\|_{2}^{2}, & \mathcal{C}=2 \sigma \kappa(\boldsymbol{p})(a+\ell \kappa(\boldsymbol{p}))\|v\|_{2}^{2}+2 \sigma \ell\left\|v_{\xi}\right\|_{2}^{2},
\end{array}\right\}
$$

where

$$
\kappa(\boldsymbol{p})=-\frac{(b+2 \sigma a \ell)}{2\left(1+\sigma \ell^{2}\right)} \quad \text { and } \quad\|v\|_{2}^{2}=\int_{-\infty}^{+\infty}|v(\xi)|^{2} \mathrm{~d} \xi .
$$

When $\boldsymbol{p} \neq 0$, it is apparent from these expressions that the stability quartic no longer has an elementary factorization, and so the stability possibilities of the deformed BSW will be more complex.

In this paper, we will restrict attention to illuminating the underlying geometry associated with long-wave transverse instability. The analysis is not rigorous, but there do not appear to be substantial obstacles to rigour. With an appropriate choice of function space, the instability results in $\S 3$ may be rigorously justifiable using a Lyapunov-Schmidt decomposition.

An outline of the paper is as follows. NLS models are Hamiltonian PDEs, and in $\S 2$ it is shown that the multi-symplectic form of Hamiltonian PDEs is the natural setting for the analysis. In this setting, the deformed BSWs are characterized by a constrained variational principle. In $\S 3$ we establish the connection between the diagnostic functionals $\mathcal{P}, \mathcal{A}, \mathcal{B}$ and $\mathcal{C}$ and the linear stability exponents. In $\S 4$ the implications of the theory for BSWs in $\chi^{(2)}$ media are discussed. 


\section{Multi-symplectifying NLS equations}

The above functionals and their relation with transverse instability will arise in a natural way when an NLS equation is formulated as a multi-symplectic Hamiltonian PDE. To illustrate, consider (1.1) and introduce new coordinates $Z=$ $\left(q_{1}, q_{2}, q_{3}, q_{4}, p_{1}, p_{2}, p_{3}, p_{4}\right) \in \mathbb{R}^{8}$, with $q_{1}+\mathrm{i} q_{2}=\psi, q_{3}+\mathrm{i} q_{4}=-\sigma\left(q_{1}+\mathrm{i} q_{2}\right)_{y}-$ $\sigma\left(p_{3}+\mathrm{i} p_{4}\right)_{x}$ and

$$
p_{1}+\mathrm{i} p_{2}=\left(q_{1}+\mathrm{i} q_{2}\right)_{x}-\sigma\left(p_{3}+\mathrm{i} p_{4}\right)_{y} .
$$

Then the NLS model (1.1) has the multi-symplectic formulation

$$
\boldsymbol{M} Z_{t}+\boldsymbol{K} Z_{x}+\boldsymbol{L} Z_{y}=\nabla S(Z), \quad Z \in \mathbb{R}^{8},
$$

with

$$
\boldsymbol{K}=\left[\begin{array}{cccc}
\mathbf{0} & \mathbf{0} & -\boldsymbol{I} & \mathbf{0} \\
\mathbf{0} & \mathbf{0} & \mathbf{0} & -\boldsymbol{I} \\
\boldsymbol{I} & \mathbf{0} & \mathbf{0} & \mathbf{0} \\
\mathbf{0} & \boldsymbol{I} & \mathbf{0} & \mathbf{0}
\end{array}\right], \quad \boldsymbol{L}=\left[\begin{array}{cccc}
\mathbf{0} & \boldsymbol{I} & \mathbf{0} & \mathbf{0} \\
-\boldsymbol{I} & \mathbf{0} & \mathbf{0} & \mathbf{0} \\
\mathbf{0} & \mathbf{0} & \mathbf{0} & -\sigma \boldsymbol{I} \\
\mathbf{0} & \mathbf{0} & \sigma \boldsymbol{I} & \mathbf{0}
\end{array}\right],
$$

$\boldsymbol{M}=\operatorname{diag}\left[-\boldsymbol{J}_{2}, \mathbf{0}, \mathbf{0}, \mathbf{0}\right]$, with $\boldsymbol{J}_{2}=\left(\begin{array}{cc}0 & -1 \\ 1 & 0\end{array}\right)$, and

$$
S(Z)=\frac{1}{2}\left(p_{1}^{2}+p_{2}^{2}+F\left(q_{1}^{2}+q_{2}^{2}\right)\right)+\frac{1}{2 \sigma}\left(q_{3}^{2}+q_{4}^{2}\right) .
$$

The system is multi-symplectic in the sense that there are distinct symplectic structures (defined by the skew-symmetric operators $\boldsymbol{M}, \boldsymbol{K}$ and $\boldsymbol{L}$ ) in the $t$-, $x$ - and $y$-directions (Bridges 1997). It is straightforward to generalize this multisymplectification to vector NLS equations. For example, general coupled NLS systems such as (1.2) are multi-symplectified in $\S 11$ of Bridges \& Derks (2001).

In (2.1), it is the transverse symplectic structure $\boldsymbol{L}$ that leads to crucial new information about susceptibility to transverse instability.

Let $G_{\varepsilon}=\operatorname{diag}\left[R_{\varepsilon}, R_{\varepsilon}, R_{\varepsilon}, R_{\varepsilon}\right]$, where $R_{\varepsilon}$ is the standard action of $S O(2)$ on $\mathbb{R}^{2}$, then the $S O(2)$ symmetry of (1.1) is represented by the fact that $\boldsymbol{M}, \boldsymbol{K}$ and $\boldsymbol{L}$ commute with $G_{\varepsilon}$ and $S\left(G_{\varepsilon} Z\right)=S(Z)$. Let $\mathfrak{g}=\left.(\mathrm{d} / \mathrm{d} \theta) G_{\theta}\right|_{\theta=0}$.

The theory can be developed in general, starting from any multi-symplectic system of the form (2.1) with an $S O(2)$ symmetry and a phase space $\mathbb{R}^{2 n}$. A deformed BSW is represented in this setting by

$$
Z(x, y, t)=G_{\theta} \hat{Z}(\xi),
$$

where $\theta$ and $\xi$ take the form (1.5) and $\hat{Z}$ satisfies

$$
\nabla S(\hat{Z})=\omega \boldsymbol{M} \mathfrak{g} \hat{Z}+a \boldsymbol{L} \mathfrak{g} \hat{Z}+b \boldsymbol{M} \hat{Z}_{\xi}+\ell \boldsymbol{L} \hat{Z}_{\xi} .
$$

The right-hand side can be characterized as the gradient of a functional; let

$$
\left.\begin{array}{rlrl}
\mathcal{P}(Z) & =\int_{\mathbb{R}} \frac{1}{2}\langle\boldsymbol{M} \mathfrak{g} Z, Z\rangle \mathrm{d} \xi, & \mathcal{A}(Z)=\int_{R} \frac{1}{2}\langle\boldsymbol{L} \mathfrak{g} Z, Z\rangle \mathrm{d} \xi, \\
\mathcal{B}(Z)=\int_{\mathbb{R}} \frac{1}{2}\left\langle\boldsymbol{M} Z_{\xi}, Z\right\rangle \mathrm{d} \xi, & \mathcal{C}(Z)=\int_{\mathbb{R}} \frac{1}{2}\left\langle\boldsymbol{L} Z_{\xi}, Z\right\rangle \mathrm{d} \xi .
\end{array}\right\}
$$

Then $\hat{Z}$ satisfies

$$
\nabla \mathcal{S}(\hat{Z})=\omega \nabla \mathcal{P}(\hat{Z})+a \nabla \mathcal{A}(\hat{Z})+b \nabla \mathcal{B}(\hat{Z})+\ell \nabla \mathcal{C}(\hat{Z}),
$$

where $\mathcal{S}(\hat{Z})$ is the integral over $\xi$ of $S(\hat{Z})$.

Proc. R. Soc. Lond. A (2004) 
For the case of (1.1), when the multi-symplectic coordinates are transformed back to $\psi$ coordinates, the above four functionals (2.2) reduce to (1.6).

The above result shows that the deformed bright solitary waves can be characterized by a constrained variational principle, with $\omega, a, b$ and $\ell$ appearing as Lagrange multipliers. It follows from standard Lagrange multiplier theory that this variational principal is non-degenerate precisely when

$$
\operatorname{det}\left[\begin{array}{llll}
\mathcal{P}_{\omega} & \mathcal{P}_{a} & \mathcal{P}_{b} & \mathcal{P}_{\ell} \\
\mathcal{A}_{\omega} & \mathcal{A}_{a} & \mathcal{A}_{b} & \mathcal{A}_{\ell} \\
\mathcal{B}_{\omega} & \mathcal{B}_{a} & \mathcal{B}_{b} & \mathcal{B}_{\ell} \\
\mathcal{C}_{\omega} & \mathcal{C}_{a} & \mathcal{C}_{b} & \mathcal{C}_{\ell}
\end{array}\right] \neq 0 .
$$

It also follows from Lagrange multiplier theory that the matrix in (2.4) is symmetric.

\section{Stability analysis of bright solitons}

Let $Z(x, y, t)=G_{\theta}(\hat{Z}(\xi)+\Upsilon(\xi, y, t))$, substitute into the system (2.1), and linearize about the basic state $\hat{Z}(\xi)$. Then, letting $\Upsilon=\operatorname{Re}\left(\mathcal{U}(\xi) \mathrm{e}^{\lambda t+\mathrm{i} \beta y}\right)$, where $\beta$ is the transverse wavenumber and $\lambda$ is the stability exponent, leads to the linearized stability equation

$$
\mathcal{L U}=\lambda \boldsymbol{M U}+\mathrm{i} \beta \boldsymbol{L U},
$$

where $\mathcal{L}=D^{2} \mathcal{S}-\omega D^{2} \mathcal{P}-a D^{2} \mathcal{A}-b D^{2} \mathcal{B}-\ell D^{2} \mathcal{C}$.

The kernel of $\mathcal{L}$ has (at least) two elements: $\mathfrak{g} \hat{Z}(\xi)$ (the tangent vector to the $G_{\theta}$ group action) and $\hat{Z}_{\xi}$. Assume that these are the only elements in the kernel (generically satisfied). Then look for long-wave transverse instabilities $|\beta| \ll 1$ with small growth rate $|\lambda| \ll 1$, by expanding $\mathcal{U}$ in a Taylor series. Consider the ansatz

$$
\mathcal{U}=c_{1}\left(\mathfrak{g} \hat{Z}+\lambda \hat{Z}_{\omega}+\mathrm{i} \beta \hat{Z}_{a}\right)+c_{2}\left(\hat{Z}_{\xi}+\lambda \hat{Z}_{b}+\mathrm{i} \beta \hat{Z}_{\ell}\right)+\mathcal{O}\left(|\lambda|^{2}+|\beta|^{2}\right),
$$

where $\boldsymbol{c}=\left(c_{1}, c_{2}\right)$ are arbitrary complex constants. By differentiating (2.3) with respect to $\omega, b, a$ and $\ell$ it can be verified that this expression is indeed the solution to (3.1) to leading order.

It is worth remarking here that it is precisely in the leading-order expression for $\mathcal{U}$ that the deformation (1.4), (1.5) is necessary. The derivatives $\hat{Z}_{a}, \hat{Z}_{b}, \hat{Z}_{\ell}$ are not necessarily needed for the stability of classical BSWs $(\boldsymbol{p}=0)$, but their limit $\boldsymbol{p} \rightarrow 0$ is required.

Since $\mathcal{L}$ is formally self-adjoint, the solvability condition for (3.1) is

$$
\begin{gathered}
\int_{\mathbb{R}}\langle\mathfrak{g} \hat{Z}, \lambda \boldsymbol{M U}+\mathrm{i} \beta \boldsymbol{L U}\rangle \mathrm{d} \xi=0, \\
\int_{\mathbb{R}}\left\langle\hat{Z}_{\xi}, \lambda \boldsymbol{M U}+\mathrm{i} \beta \boldsymbol{L U}\right\rangle \mathrm{d} \xi=0,
\end{gathered}
$$

where $\langle\cdot, \cdot\rangle$ is the inner product on the (complexified) phase space for (2.1). Substituting the leading-order expression for $\mathcal{U}$ into the solvability condition leads to the pair of algebraic equations

$$
\left[\boldsymbol{N}_{0} \lambda^{2}+\mathrm{i} \beta \lambda \boldsymbol{N}_{1}+(\mathrm{i} \beta)^{2} \boldsymbol{N}_{2}+\cdots\right]\left(\begin{array}{l}
c_{1} \\
c_{2}
\end{array}\right)=\left(\begin{array}{l}
0 \\
0
\end{array}\right),
$$

Proc. R. Soc. Lond. A (2004) 
where $\boldsymbol{N}_{j}, j=0,1,2$, are $2 \times 2$ matrices depending only on the properties of the basic solitary wave. The derivation of the expression for $\boldsymbol{N}_{0}$ will be given, and then the result for the other two will be stated. From the solvability condition we have that

$$
\boldsymbol{N}_{0}=\left[\begin{array}{ll}
\int_{\mathbb{R}}\left\langle\mathfrak{g} \hat{Z}, \boldsymbol{M} \hat{Z}_{\omega}\right\rangle \mathrm{d} \xi & \int_{\mathbb{R}}\left\langle\mathfrak{g} \hat{Z}, \boldsymbol{M} \hat{Z}_{b}\right\rangle \mathrm{d} \xi \\
\int_{\mathbb{R}}\left\langle\hat{Z}_{\xi}, \boldsymbol{M} \hat{Z}_{\omega}\right\rangle \mathrm{d} \xi & \int_{\mathbb{R}}\left\langle\hat{Z}_{\xi}, \boldsymbol{M} \hat{Z}_{b}\right\rangle \mathrm{d} \xi
\end{array}\right] .
$$

However, by differentiating the functionals (2.2) with respect to $b$ and $\omega$ we find that the matrix simplifies to

$$
\boldsymbol{N}_{0}=-\left[\begin{array}{ll}
\frac{\partial \mathcal{P}}{\partial \omega} & \frac{\partial \mathcal{P}}{\partial b} \\
\frac{\partial \mathcal{B}}{\partial \omega} & \frac{\partial \mathcal{B}}{\partial b}
\end{array}\right]
$$

Similarly,

$$
\boldsymbol{N}_{1}=-\left[\begin{array}{ll}
\frac{\partial \mathcal{P}}{\partial a}+\frac{\partial \mathcal{A}}{\partial \omega} & \frac{\partial \mathcal{P}}{\partial \ell}+\frac{\partial \mathcal{A}}{\partial b} \\
\frac{\partial \mathcal{B}}{\partial a}+\frac{\partial \mathcal{C}}{\partial \omega} & \frac{\partial \mathcal{B}}{\partial \ell}+\frac{\partial \mathcal{C}}{\partial b}
\end{array}\right] \quad \text { and } \quad \boldsymbol{N}_{2}=-\left[\begin{array}{ll}
\frac{\partial \mathcal{A}}{\partial a} & \frac{\partial \mathcal{A}}{\partial \ell} \\
\frac{\partial \mathcal{C}}{\partial a} & \frac{\partial \mathcal{C}}{\partial \ell}
\end{array}\right] .
$$

Neglecting the higher-order terms in $\lambda$ and $\beta$, the basic result is that all long-wave transverse instabilities with small growth rate are determined by the roots of the quartic

$$
0=\operatorname{det}\left[\boldsymbol{N}_{0} \lambda^{2}+\mathrm{i} \beta \lambda \boldsymbol{N}_{1}+(\mathrm{i} \beta)^{2} \boldsymbol{N}_{2}\right]=\beta^{4} \Delta(\Omega),
$$

by letting $\lambda=\mathrm{i} \Omega \beta$, where $\Delta(\Omega)$ is defined in (1.7).

If there exists a root of $\Delta(\Omega)=0$ with non-zero imaginary part, the basic state is transverse unstable. It would appear from the definition $\lambda=\mathrm{i} \Omega \beta$ that $\operatorname{Im}(\Omega)<0$ would be required for instability. However, due to the Hamiltonian symmetry, if there exists a root with positive imaginary part, there will exist one with negative imaginary part. This follows because coupled with (3.3) is

$$
\left[\boldsymbol{N}_{0} \lambda^{2}-\mathrm{i} \beta \lambda \boldsymbol{N}_{1}+(\mathrm{i} \beta)^{2} \boldsymbol{N}_{2}+\cdots\right]\left(\begin{array}{l}
d_{1} \\
d_{2}
\end{array}\right)=\left(\begin{array}{l}
0 \\
0
\end{array}\right)
$$

associated with the mode proportional to $\mathrm{e}^{-\mathrm{i} \beta y}$. Hence given a stability exponent $\lambda(\beta)$ there exists a mode $\lambda(-\beta)$. Another way to see this is to let

$$
\Upsilon(x, y, t)=\mathrm{e}^{\lambda t}\left(\mathcal{V}_{1}(\xi) \cos \beta y+\mathcal{V}_{2}(\xi) \sin \beta y\right) .
$$

Then the system (3.1) becomes the coupled system

$$
\mathcal{L} \mathcal{V}_{1}=\lambda \boldsymbol{M} \mathcal{V}_{1}+\beta \boldsymbol{L} \mathcal{V}_{2} \quad \text { and } \quad \mathcal{L V}_{2}=\lambda \boldsymbol{M} \mathcal{V}_{2}-\beta \boldsymbol{L} \mathcal{V}_{1}
$$

An expansion of the type (3.2) can then be obtained for $\mathcal{V}_{1}$ and $\mathcal{V}_{2}$ with four coefficients, say $c_{1}, c_{2}, c_{3}, c_{4}$, leading to four equations instead of (3.3). But the four equations can be block diagonalized leading to the $c_{1}, c_{2}$ plus $d_{1}, d_{2}$ systems above.

The instability condition is a sufficient condition for long-wave transverse instability. The converse - all roots of $\Delta(\Omega)=0$ real-is a necessary but not sufficient 
condition for stability. There are two ways that a wave can be transverse unstable when all roots of $\Delta(\Omega)=0$ are real: either a short-wave instability $(|\beta|$ finite), or $|\beta|$ small but $|\lambda|$ finite.

\section{(a) Simplification of the stability quartic for scalar NLS equations}

All long-wave instabilities with $|\lambda| \ll 1$ of the deformed solitary waves are determined by the roots of the quartic (3.4). However, for classical BSWs, the quartic has an elementary factorization.

First, a solution of the form (1.4) of (1.1) which reduces to a classical BSW when $\boldsymbol{p} \rightarrow 0$ has the form

$$
\psi(x, y, t)=\mathrm{e}^{\mathrm{i} \theta} \mathrm{e}^{\mathrm{i} \kappa(\boldsymbol{p}) \xi} v(\xi),
$$

where $v(\xi)$ is now real valued (and depends on $\boldsymbol{p}$ ) and $\kappa(\boldsymbol{p})$ is defined in (1.11). With this form for $\psi$, the four diagnostic functionals reduce to the form (1.10). The key functional here is $\mathcal{B}$. Since $\kappa(\boldsymbol{p})=0$ when $\boldsymbol{p}=0$, it follows that

$$
\mathcal{B}_{\omega}^{0}=0, \quad \mathcal{B}_{a}^{0}=0, \quad \mathcal{B}_{\ell}^{0}=0 \quad \text { and } \quad \mathcal{B}_{b}^{0}=-\frac{1}{2}\|u\|_{2}^{2},
$$

where the superscript ' 0 ' indicates evaluation at $\boldsymbol{p}=0$ and $\|u\|_{2}$ is the $L_{2}(\mathbb{R})$ norm of the classical BSW.

Proceeding this way using (1.10), one shows that the only non-zero elements in the stability quartic are the diagonal entries in the Jacobian (2.4) and they are

$$
\mathcal{P}_{\omega}^{0}=\partial_{\omega}\|u\|_{2}^{2}, \quad \mathcal{A}_{a}^{0}=2 \sigma\|u\|_{2}^{2}, \quad \mathcal{B}_{b}^{0}=-\frac{1}{2}\|u\|_{2}^{2}, \quad \mathcal{C}_{\ell}^{0}=2 \sigma\left\|u_{x}\right\|_{2}^{2} .
$$

It is immediate from the criteria (1.8) that classical BSWs are unstable if $\sigma<0$ and if $\sigma>0$ they are unstable if $\mathcal{P}_{\omega}^{0}>0$. This result recovers the classical result on the long-wave transverse instability of BSWs (Zakharov \& Rubenchik 1973; see also Kuznetsov et al. 1986; Rasmussen \& Rypdal 1986; Kivshar \& Pelinovsky 2000).

\section{Bright solitons in $\chi^{(2)}$ or mixed $\chi^{(2)}-\chi^{(3)}$ media}

There is considerable interest in the existence and stability of bright solitary waves in media governed by NLS equations including a quadratic nonlinearity (e.g. Bang et al. 1998; Kivshar \& Pelinovsky 2000; Liu et al. 2000; Parker 1998; Yew et al. 1999; and references therein). In this section, we show that the transverse instability theory of this paper applies directly to these models. We will use the mixed $\chi^{(2)}-\chi^{(3)}$ model of Bang et al. for illustration.

By multi-symplectifying (1.2), the diagnostic functionals can be determined. Transforming back to $(\psi, \varphi)$ coordinates and letting $\psi(x, y, t)=\mathrm{e}^{\mathrm{i} \theta} u(\xi)$ and $\varphi(x, y, t)=$ $\mathrm{e}^{2 \mathrm{i} \theta} v(\xi)$, they are

$$
\begin{aligned}
& \mathcal{P}=\int_{\mathbb{R}}\left(|u(\xi)|^{2}+4|v(\xi)|^{2}\right) \mathrm{d} \xi, \\
& \mathcal{B}=\int_{\mathbb{R}}\left(\operatorname{Im}\left(\bar{u} u^{\prime}\right)+2 \operatorname{Im}\left(\bar{v} v^{\prime}\right)\right) \mathrm{d} \xi, \\
& \mathcal{A}=2 \int_{\mathbb{R}}\left(\sigma_{1} a|u|^{2}+4 \sigma_{2} a|v|^{2}+\sigma_{1} \ell \operatorname{Im}\left(\bar{u} u^{\prime}\right)+2 \sigma_{2} \ell \operatorname{Im}\left(\bar{v} v^{\prime}\right)\right) \mathrm{d} \xi, \\
& \mathcal{C}=2 \int_{\mathbb{R}}\left(\sigma_{1} \ell\left|u^{\prime}\right|^{2}+\sigma_{2} \ell\left|v^{\prime}\right|^{2}+\sigma_{1} a \operatorname{Im}\left(\bar{u} u^{\prime}\right)+2 \sigma_{2} a \operatorname{Im}\left(\bar{v} v^{\prime}\right)\right) \mathrm{d} \xi .
\end{aligned}
$$

Proc. R. Soc. Lond. A (2004) 
By restricting attention to classical BSWs, some general conclusions can be drawn. By differentiating and then taking the limit as $\boldsymbol{p} \rightarrow 0$, we find that

$$
\begin{aligned}
& \mathcal{P}_{\omega}^{0}=\partial_{\omega} \int_{-\infty}^{+\infty}\left[u(x)^{2}+4 v(x)^{2}\right] \mathrm{d} x, \\
& \mathcal{B}_{b}^{0}=\lim _{p \rightarrow 0} \int_{-\infty}^{+\infty} \frac{1}{b}\left(\operatorname{Im}\left(\bar{u} u^{\prime}\right)+2 \operatorname{Im}\left(\bar{v} v^{\prime}\right)\right) \mathrm{d} x \\
& \mathcal{A}_{a}^{0}=\int_{-\infty}^{+\infty}\left[2 \sigma_{1} u(x)^{2}+8 \sigma_{2} v(x)^{2}\right] \mathrm{d} x \\
& \mathcal{C}_{\ell}^{0}=\int_{-\infty}^{+\infty}\left[2 \sigma_{1} u^{\prime}(x)^{2}+2 \sigma_{2} v^{\prime}(x)^{2}\right] \mathrm{d} x .
\end{aligned}
$$

The first functional derivative is the derivative of the power used in Bang et al. (1998) when applying the VK condition to BSWs in mixed media. Bang et al. observed that the VK condition is not conclusive regarding longitudinal instability. Normally $\mathcal{P}_{\omega}>0$ implies longitudinal stability, but they find a case where $\mathcal{P}_{\omega}>0$ waves are longitudinally unstable.

The problem with the VK condition for longitudinal instability is that one must check the spectrum of the second variation of the Lagrange functional $H-\omega P$ before one can ensure that the VK condition is conclusive. On the other hand, for transverse instability the VK derivative can provide conclusive information: as shown below, when $\mathcal{P}_{\omega}>0$ the wave is transverse unstable, and this conclusion is obtained independent of the spectrum of the second variation of the Lagrange functional $S-\omega P+\cdots$.

For example, suppose $\sigma_{1}$ and $\sigma_{2}$ are both of the same sign. Then the bright solitary waves are (long-wave) transverse unstable if $\sigma_{1} P_{\omega}>0$. If $\sigma_{1} P_{\omega}<0$, the bright solitary waves (1.4) of (1.2) are transverse unstable if

$$
\sigma_{1} \mathcal{B}_{b}^{0}=\sigma_{1} \lim _{p \rightarrow 0} \int_{-\infty}^{+\infty} \frac{1}{b}\left(\operatorname{Im}\left(\bar{u} u^{\prime}\right)+2 \operatorname{Im}\left(\bar{v} v^{\prime}\right)\right) \mathrm{d} x>0 .
$$

Now suppose $\sigma_{1}=\sigma_{2}=\sigma$. Then it can be shown explicitly that $\mathcal{B}_{b}^{0}=-\frac{1}{2} \mathcal{P}^{0}$. In this case, if $\sigma<0$ the BSWs are transverse unstable, but if $\sigma>0$ they are transverse unstable if $\mathcal{P}_{\omega}>0$, independent of whether they are longitudinally stable or unstable. In Bang et al. the parameters $\sigma_{1}$ and $\sigma_{2}$ are equal and positive. Therefore, in their model, long-wave transverse instability of $\boldsymbol{p}=0$ BSWs occurs only if $\mathcal{P}_{\omega}>0$. One can therefore read off their fig. 8 (which shows $\mathcal{P}$ as a function of $\omega$ ) points of transverse instability using the above condition.

In models where $\sigma_{1}$ and $\sigma_{2}$ have opposite sign, many other possibilities for instability arise, depending on the relative power of the two components $u$ and $v$ of the wave.

\section{Concluding remarks}

With modest assumptions, the theory applies to any NLS-type model that can be multi-symplectified and has an $S O(2)$ symmetry.

There are a number of directions for generalization of the result in this paper. One direction is to consider transverse instability of soliton solutions of NLS models 
in inhomogeneous media. A prototype is the Kronig-Penney model (Gaididei et al. 1997). The new issue with inhomogeneous media is that momentum is no longer conserved.

Another interesting generalization is the case of transverse instability of vector solitons of coupled NLS equations with $S O(2) \times S O(2)$ symmetry, or, more generally, $N$ coupled NLS equations with $S O(2)^{N}$ symmetry. For example, the generalization of the deformation theory presented here to coupled NLS equations with $S O(2) \times S O(2)$ symmetry can be sketched as follows. Taking (1.2) as an example, if the quadratic terms are absent and the nonlinearity is cubic, the system has $S O(2) \times S O(2)$ symmetry and the bright solitary waves (1.4) are generalized to

$$
\psi(x, t)=\mathrm{e}^{\mathrm{i} \omega_{1} t} u(x), \quad \varphi(x, t)=\mathrm{e}^{\mathrm{i} \omega_{2} t} v(x),
$$

with $u(x)$ and $v(x)$ real valued, and $\omega_{1}$ not equal to $\omega_{2}$ in general. The required deformation is then six dimensional,

$$
\psi(x, y, t)=\mathrm{e}^{\mathrm{i} \theta_{1}} u(\xi) \text { and } \varphi(x, y, t)=\mathrm{e}^{\mathrm{i} \theta_{2}} v(\xi),
$$

where now $\theta_{j}=\omega_{j} t+a_{j} y+\theta_{j}^{0}$. The multi-symplectification will generate six diagnostic functionals, the kernel of the operator $\mathcal{L}$ in (3.1) will have three elements and the stability quartic will become a polynomial of degree 6 . A similar strategy can be used to generalize to $n$ coupled NLS equations with $S O(2)^{n}$ symmetry. The full implications of these generalizations will be considered elsewhere.

\section{References}

Bang, O., Kivshar, Yu. S., Buryak, A. V., DeRossi, A. \& Trillo, S. 1998 Two-dimensional solitary waves in media with quadratic and cubic nonlinearity. Phys. Rev. E 58, 5057-5069.

Bridges, T. J. 1997 Multi-symplectic structures and wave propagation. Math. Proc. Camb. Phil. Soc. 121, 147-190.

Bridges, T. J. 2000 Universal geometric condition for the transverse instability of solitary waves. Phys. Rev. Lett. 84, 2614-2617.

Bridges, T. J. \& Derks, G. 2001 The symplectic Evans matrix, and the instability of solitary waves and fronts with symmetry. Arch. Ration. Mech. Analysis 156, 1-87.

Gaididei, Yu. B., Christiansen, P. L., Rasmussen, K. O. \& Johansson, M. 1997 Two-dimensional effects in nonlinear Kronig-Penney models. Phys. Rev. B 55, R13 365-R13 368.

Grillakis, M., Shatah, J. \& Strauss, W. A. 1987 Stability theory of solitary waves in the presence of symmetry. J. Funct. Analysis 74, 160-197.

Kivshar, Yu. S. \& Pelinovsky, D. E. 2000 Self-focusing and transverse instabilities of solitary waves. Phys. Rep. 331, 117-195.

Kuznetsov, E. A., Rubenchik, A. M. \& Zakharov, V. E. 1986 Soliton stability in plasmas and hydrodynamics. Phys. Rep. 142, 103-165.

Liu, X., Beckwitt, K. \& Wise, F. 2000 Transverse instability of optical spatiotemporal solitons in quadratic media. Phys. Rev. Lett. 85, 1871-1874.

Parker, D. F. 1998 Exact representations for coupled bright and dark solitary waves of quadratically nonlinear systems. J. Opt. Soc. Am. B 15, 1061-1068.

Rasmussen, J. J. \& Rypdal, K. 1986 Blow-up in nonlinear Schrödinger equations. I. A general review. Physica Scr. 33, 481-497.

Vakhitov, N. G. \& Kolokolov, A. A. 1973 Stationary solutions of the wave equation in media with nonlinearity saturation. Radiophys. Quant. Electron. 16, 1020-1028.

Proc. R. Soc. Lond. A (2004) 
Weinstein, M. I. 1986 Lyapunov stability of ground states of nonlinear dispersive evolution equations. Commun. Pure Appl. Math. 39, 51-76.

Yew, A. C., Champneys, A. R. \& McKenna, P. J. 1999 Multiple solitary waves due to secondharmonic generation in quadratic media. J. Nonlin. Sci. 9, 33-52.

Zakharov, V. E. \& Rubenchik, A. M. 1973 Instability of waveguides and solitons in nonlinear media. Z. Eksperiment. Teoret. Fiz. 65, 997-1011. 
